\title{
Commentary: Impact of the COVID-19 Pandemic on the Mental Health of College Students: A Systematic Review and Meta-Analysis
}

\author{
Joel Swai ${ }^{1,2 *}$, Adam Mohamed ${ }^{3}$ and Jing-ping Zhang ${ }^{4}$ \\ ${ }^{1}$ Department of Medicine, University of Alberta, Edmonton, AB, Canada, ${ }^{2}$ Department of Internal Medicine, Benjamin Mkapa \\ Hospital, Dodoma, Tanzania, ${ }^{3}$ Mental Health Institute of the Second Xiangya Hospital, Central South University, Changsha, \\ China, ${ }^{4}$ Department of Nursing Management, Xiangya School of Nursing, Central South University, Changsha, China
}

Keywords: college students, coronavirus disease 2019, mental health, depression, anxiety, meta-analysis

\section{A Commentary on}

Impact of the COVID-19 Pandemic on the Mental Health of College Students: A Systematic Review and Meta-Analysis

OPEN ACCESS

Edited by:

Stephen Clift,

Canterbury Christ Church University,

United Kingdom

Reviewed by:

Aleksandra Maria Rogowska,

University of Opole, Poland

*Correspondence:

Joel Swai

jswai@ualberta.ca

orcid.org/0000-0001-5363-3977

Specialty section:

This article was submitted to

Health Psychology,

a section of the journal

Frontiers in Psychology

Received: 05 August 2021

Accepted: 25 October 2021

Published: 16 November 2021

Citation:

Swai J, Mohamed A and Zhang J-p (2021) Commentary: Impact of the COVID-19 Pandemic on the Mental

Health of College Students: A

Systematic Review and Meta-Analysis.

Front. Psychol. 12:753798.

doi: 10.3389/fpsyg.2021.753798 by Li, Y., Wang, A., Wu, Y., Han, N., and Huang, H. (2021). Front. Psychol. 12:669119. doi: 10.3389/fpsyg.2021.669119

\section{INTRODUCTION}

Coronavirus Disease 2019 (COVID-19) pandemic has resulted in adverse psychological effects among individuals globally. Recurring infection waves, extended lockdowns, the emergence of more transmissible, and immunity evasive variants are among the unpredictable factors that lead to an increased sense of fear associated with the pandemic (Batista et al., 2021). Moreover, social isolation, quarantines, unemployment, and long-covid are strongly associated with depression (Loades et al., 2020; Islam et al., 2021). These issues indicate the need for epidemiological studies exploring the pandemic's effect on mental health.

In a recent meta-analysis study by Li et al. (2021), the authors pooled the prevalence of anxiety and depression associated with COVID-19 among college students. They reported a prevalence of 36\% [95\% Confidence Interval (CI): 26-46] and 39\% [CI: 27-51] for anxiety and depression, respectively, both with statistically significant high heterogeneity of $99.9 \%, p<0.01$. In their study limitations section, authors called upon further heterogeneity analyses by considering the confounding factors. In response, we performed a meta-regression and pooled the prevalence of anxiety and depression stratifying results by potential confounders (i.e., gender, survey instrument used, survey country, and survey dates).

\section{PREVALENCE OF ANXIETY}

Figures 1A-D summarizes the meta-regression of pooled proportions (Proportion = prevalence/100\%) of anxiety stratified by survey date, survey instrument, survey country, and male-female ratio. The prevalence of anxiety was statistically significant higher in studies conducted after March the first [beta $=2.68$; standard error $(\mathrm{SE})=0.67, t=3.95, p=0.001$; CI: $1.58-4.55]$. There was no statistically significant difference in the prevalence among studies using 


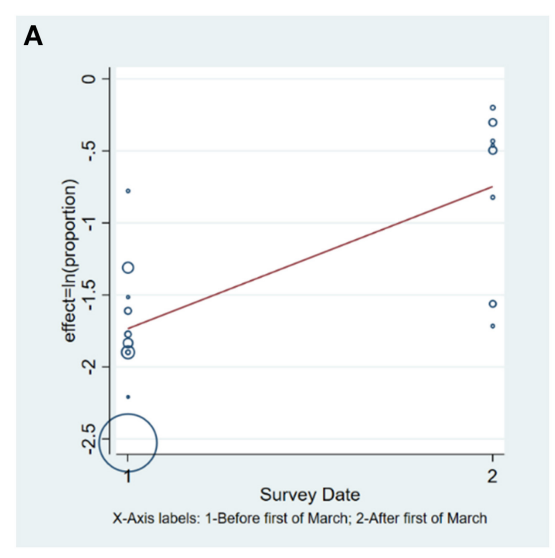

B

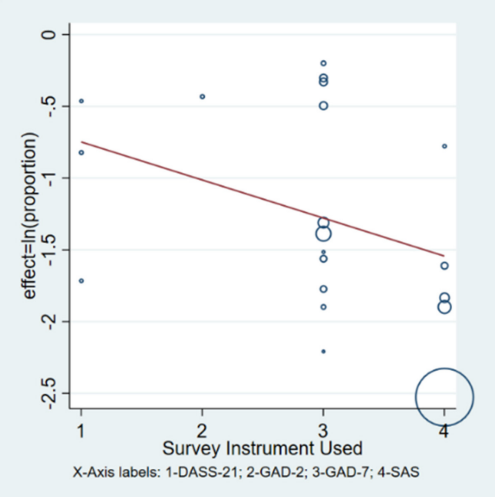

D

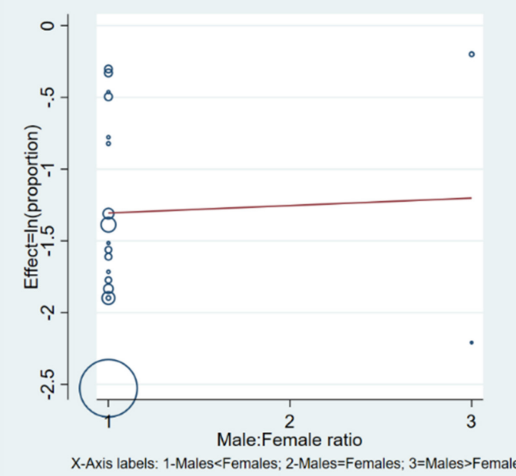

G

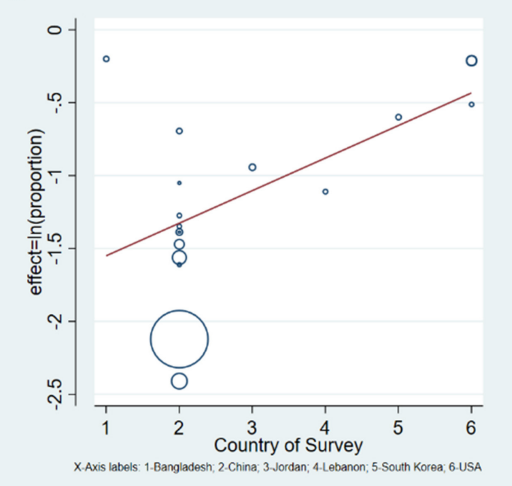

E

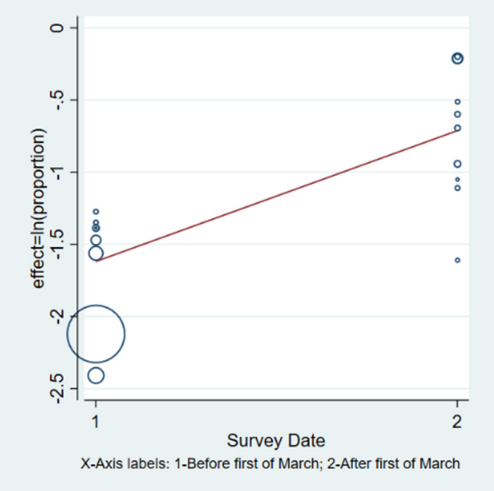

H

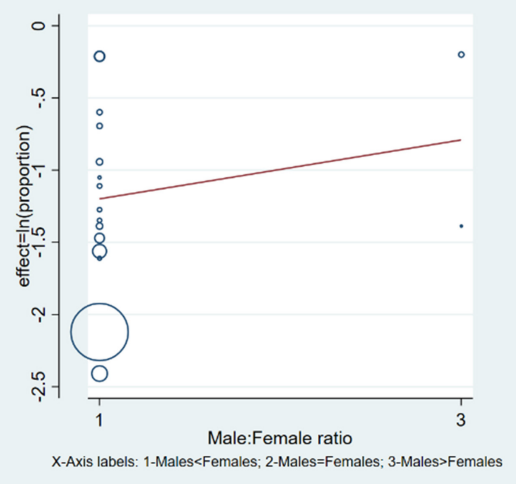

C

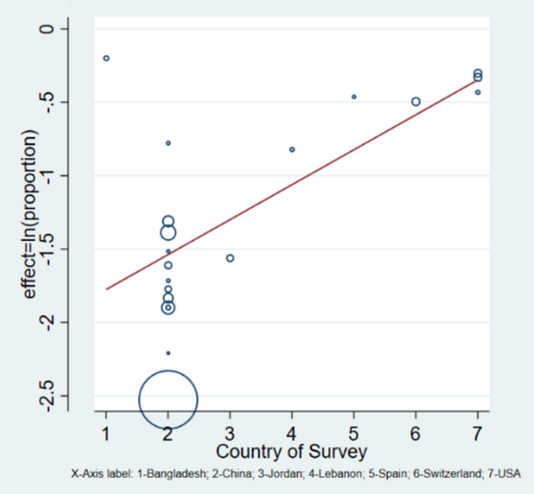

F

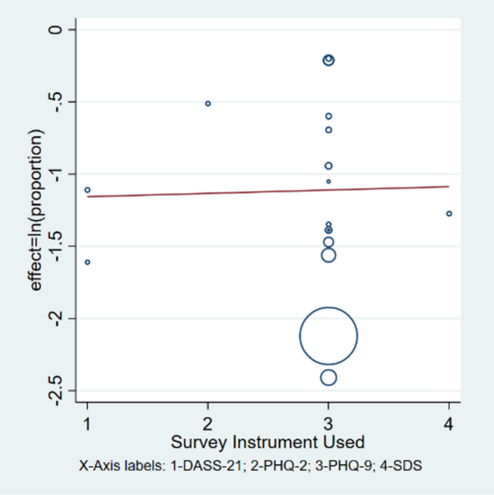

FIGURE 1 | Meta-regression of pooled proportions (proportion = prevalence/100\%) of anxiety and depression. (A) Anxiety proportion stratified by survey date; (B) anxiety proportion stratified by survey instrument; (C) anxiety proportion stratified by survey country; (D) anxiety proportion stratified by male-female ratio; (E) depression proportion stratified by survey dates; (F) depression proportion stratified by survey instrument; (G) depression proportion stratified by survey country; and (H) depression proportion stratified by male-female ratio.

different survey instruments (i.e., DASS, GAD-2, GAD-7, or SAS) to assess anxiety [beta $=0.77 ; \mathrm{SE}=0.12, t=-1.64, p=0.119$; CI: 0.55-1.08].

Moreover, the prevalence of anxiety in Asian (i.e., Bangladesh, China, and Jordan) countries were lower as compared to European (i.e., Spain, Switzerland) and America [beta $=1.27$; SE $=0.08, t=3.86, p=0.001 ; \mathrm{CI}$ : 1.11-1.44]. Interestingly, studies with more males than females (i.e., male: female $>1$ ) had higher prevalence of anxiety than studies with more females than males (i.e., male; females $<1$ ) [beta $=0.26$; $\mathrm{SE}=0.09, t=3.69, p=$ 0.002; CI: 0.12-0.56].

Having identified the confounders, we, therefore, pooled the overall prevalence of anxiety only including studies (Jiang et al., 2020; Liu et al., 2020; Wang and Zhao, 2020) conducted in the same country (i.e., China), on the same survey dates (i.e., before the first of March), and having the same male-female ratio 
(i.e., 0.7). The overall proportion was 0.15 [CI: 0.14-0.16] (i.e., prevalence $=15 \%$ ) with non-significant heterogeneity $\left(I^{2}\right)$ of $63.8 \%, p=0.063$. There was no sufficient data to conduct this analysis in other countries.

\section{PREVALENCE OF DEPRESSION}

Figures 1E-H illustrates the meta-regression of pooled prevalence of depression stratified by survey date, survey instrument, survey country, and male-female ratio. Studies conducted after the first of March had a higher pooled prevalence of depression than before [beta $=2.48$; SE $=0.52, t=4.31, p=0.001 ; \mathrm{CI}: 1.58-3.87]$. There was no statistically significantly difference in depression prevalence among different studies that used separate survey instruments [beta $=1.02 ; \mathrm{SE}=0.22, t=0.11, p=0.916$; CI: 0.65-1.62].

Studies conducted in Asia reported a statistically significantly lower prevalence of depression than studies conducted in America and South-East Asia [beta $=1.25 ; \mathrm{SE}=0.096, t=2.90$, $p=0.01$; CI: 1.06-1.47]. Again, studies with more males than females had higher prevalence of depression as compared to more females than males [beta $=0.25 ; \mathrm{SE}=0.082, t=4.19, p=0.001$; CI: $0.12-0.50]$.

\section{REFERENCES}

Batista, P., Duque, V., Luzio-Vaz, A., and Pereira, A. (2021). Anxiety impact during COVID-19: a systematic review. J. Infect. Develop. Countries 15, 320-325. doi: $10.3855 /$ jidc. 12730

Chang, J., Yuan, Y., and Wang, D. (2020). [Mental health status and its influencing factors among college students during the epidemic of COVID-19]. Nan Fang Yi Ke Da Xue Xue Bao. 40, 171-176. doi: 10.12122/j.issn.1673-4254.2020.02.06

Chi, X., Becker, B., Yu, Q., Willeit, P., Jiao, C., Huang, L., et al. (2020). Prevalence and psychosocial correlates of mental health outcomes among Chinese college students during the coronavirus disease (COVID-19) pandemic. Front. Psychiatry. 11:803. doi: 10.3389/fpsyt.2020.00803

Islam, M. S., Ferdous, M. Z., Islam, U. S., Mosaddek, A. S. M., Potenza, M. N., and Pardhan, S. (2021). Treatment, persistent symptoms, and depression in people infected with COVID-19 in Bangladesh. Int. J. Environ. Res. Public Health 18:1453. doi: 10.3390/ijerph18041453

Jiang, Y., He, L., Meng, Y., Liu, Z., Zhang, T., Zhou, Y., et al. (2020). Analysis of mental health status and influencing factors of preventive medicine students in a university during the outbreak of new coronavirus pneumonia. J. Xiangnan Univers. 22, 59-62.

Li, Y., Wang, A., Wu, Y., Han, N., and Huang, H. (2021). Impact of the COVID19 pandemic on the mental health of college students: a systematic review and meta-analysis. Front. Psychol. 12:669119. doi: 10.3389/fpsyg.2021.669119

Liu, J., Zhu, Q., Fan, W., Makamure, J., Zheng, C., and Wang, J. (2020). Online mental health survey in a medical college in china during the COVID-19 outbreak. Front. Psychiatry 11:459. doi: 10.3389/fpsyt.2020.00459
We pooled the overall prevalence of depression only including studies (Chang et al., 2020; Chi et al., 2020) conducted in the same country (i.e., China), on the same survey dates (i.e., before the first of March), having the same male-female ratio (i.e., 0.6) and used same survey instrument (i.e., PHQ-9). The overall proportion was 0.22 [CI: $0.20-0.24$ ] (i.e., prevalence $=22 \%$ ) with non-significant heterogeneity $\left(I^{2}\right)$ of $66.80 \%, p=0.083$. Other countries had no sufficient data to conduct this analysis.

\section{CONCLUSIONS}

The differences in the college students' prevalence of depression and anxiety among nations and study survey dates, reported by $\mathrm{Li}$ et al., are statistically significant (i.e., $p<0.05$ ). However, the prevalence of anxiety and depression are 15 and $22 \%$, respectively, instead of 36 and $39 \%$ reported by $\mathrm{Li}$ et al. In addition, studies with more males than females had a statistically significant higher prevalence of anxiety and depression than studies with more females than males.

\section{AUTHOR CONTRIBUTIONS}

All authors listed have made a substantial, direct and intellectual contribution to the work, and approved it for publication.

Loades, M. E., Chatburn, E., Higson-Sweeney, N., Reynolds, S., Shafran, R., Brigden, A., et al. (2020). Rapid systematic review: the impact of social isolation and loneliness on the mental health of children and adolescents in the context of COVID-19. J. Am. Acad. Child Adolesc. Psychiatry 59, 1218.e3-1239.e3. doi: $10.1016 /$ j.jaac.2020.05.009

Wang, C., and Zhao, H. (2020). The impact of COVID-19 on anxiety in Chinese university students. Front. Psychol. 11:1168. doi: 10.3389/fpsyg.2020.01168

Conflict of Interest: The authors declare that the research was conducted in the absence of any commercial or financial relationships that could be construed as a potential conflict of interest.

Publisher's Note: All claims expressed in this article are solely those of the authors and do not necessarily represent those of their affiliated organizations, or those of the publisher, the editors and the reviewers. Any product that may be evaluated in this article, or claim that may be made by its manufacturer, is not guaranteed or endorsed by the publisher.

Copyright (c) 2021 Swai, Mohamed and Zhang. This is an open-access article distributed under the terms of the Creative Commons Attribution License (CC BY). The use, distribution or reproduction in other forums is permitted, provided the original author(s) and the copyright owner(s) are credited and that the original publication in this journal is cited, in accordance with accepted academic practice. No use, distribution or reproduction is permitted which does not comply with these terms. 